\title{
P1148A in fibrillin-1 is not a mutation anymore
}

Sir - Like mutations in most heritable disorders of connective tissue, fibrillin-1 (FBN1) mutations in the spectrum of the Marfan syndrome (MFS) are most often private (see ref. 1 for review). Thus, finding an identical molecular change in more than one affected individual or family has been rare. One exception is the substitution of alanine for proline at position 1148 (P1148A) in exon 27 of FBN1. Tynan and co-workers ${ }^{2}$ were the first to report this mutation in 14 individuals, of which 6 were probands. All but one of these individuals had classic MFS (characterized by skeletal, ocular, and cardiovascular abnormalities ${ }^{3}$ ), late onset aortic aneurysms or were first degree relatives of an affected individual. One person in the control population was found to carry the 'mutation.' Sood et al. ${ }^{4}$ recently reported the expansion of the spectrum of FBN1 microfibrillopathies to include Shprintzen-Goldberg syndrome (SGS), a disorder characterized by Marfanoid habitus, craniosynostosis and mental retardation. They found $\mathrm{P} 1148 \mathrm{~A}$ in three of 60 affected families; one with SGS, one with classic MFS and one with late onset aortic aneurysm.

Here we report P1148A in five individuals from a mixed patient population (Table 1) but not in 120 Caucasian or 50 African American controls. Our screening protocol for FBN1 mutations includes heteroduplex analysis of amplicons generated from each of the 65 exons of $F B N 1$ using primers that flank each exon ${ }^{5}$. As we continued our analyses, we found additional changes consistent with mutational events in two of the five patients in whom P1148A was found (patients 496 and 503; Table 1). In the case of patient 503, sequencing of cloned RT-PCR products indicated that $\mathrm{P} 1148 \mathrm{~A}$ and the novel del5652T were in different $F B N 1$ alleles.

It was of interest that three of our patients having the P1148A allele were Japanese. The ethnicity of the other two patients was Hispanic (Mexican) and Caucasian (Russian). Furthermore, five of the six probands with $\mathrm{P} 1148 \mathrm{~A}$ reported by Tynan ${ }^{2}$ were of Asian descent, including the control individual. The ethnicity of patients analysed by Sood et al. ${ }^{4}$ was not reported. Therefore we studied 25 unrelated native Chinese individuals to determine whether P1148A is a polymorphism more commonly found in an Asian population. Eight of these individuals were P1148A heterozygotes. We found no homozygotes. These studies clearly show that P1148A is not, in and of itself, a deleterious change. The possibility that P1148A may exert a modifying affect on fibrillin mutations can and should be addressed in kindreds in which MFS and P1148A are both found.

Finally, these results confirm the hypothesis that $\mathrm{P} 1148 \mathrm{~A}$ is a polymorphic variant rather than a disease-causing mutation.
They are also a useful reminder that consideration of genetic variety between ethnic populations is essential.

Mei Wang ${ }^{1,2}$, Kurt R. Mathews', Kiyoshi Imaizumi ${ }^{3}$, Soraya Beiraghi ${ }^{1,2}$, Bruce Blumberg ${ }^{4}$, Maren Scheuner ${ }^{5}$,

John M. Graham, Jr. ${ }^{5}$ \&

Maurice Godfrey ${ }^{1,2}$

1 Munroe Center for Human Genetics, University of Nebraska Medical Center, 600 South 42nd Street, Omaha, NE 68194-5430. 2Department of Pediatrics University of Nebraska Medical Center ${ }^{3}$ Kanagawa Children's Medical Center, Yokohama, Japan. ${ }^{4}$ Permanente Medical Group, San Francisco, California. ${ }^{5} \mathrm{Cedars}$ Sinai Medical Center, Los Angeles, California. Correspondence should be addressed to M.G.

\section{Acknowledgements}

This work was supported in part by a grant from the National Heart, Lung, and Blood Institute of the National Institutes of Health HL48126 (M.G.) M.G. is an Established Investigator of the American Heart Association (funding was contributed in part by the AHA Florida Affiliate).

Dietz, H.C. \& Pyeritz, R.E. Human Molec. Genet. 4, 1799-1809 (1995)

2. Tynan, K. et al. Hum. Mol. Genet. 2, 1813-1821 (1993).

3. Godfrey, M. The Marfan syndrome. in McKusick's heritable disorders of connective tissue (ed. Beighton, P.) 51-135 (The C.V. Mosby Company, St. Louis, 1993).

4. Sood, S., Eldadah, Z.A., Krause, W.L., Melntosh, I. \& Dietz, H.C. Nat. Genet. 12, 209-211 (1996).

5. Nijbroek, G. et al. Am. J. Hum. Genet. 57, 8-21 (1995)

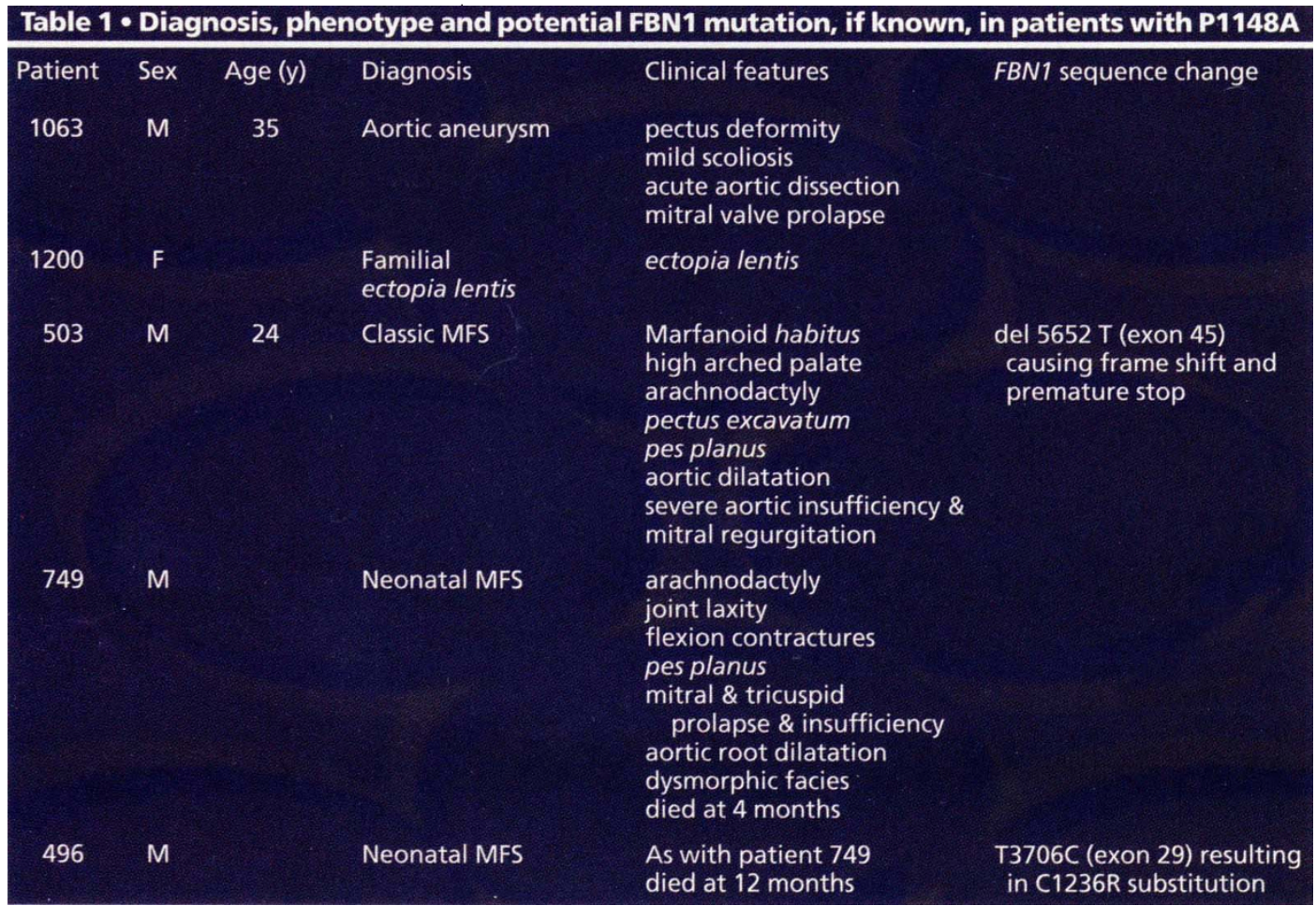

